\title{
XMM-Newton observation of the persistent Be/NS X-ray binary pulsar RX J0440.9+4431
}

\author{
N. La Palombara ${ }^{1}$, L. Sidoli ${ }^{1}$, P. Esposito ${ }^{2}$, A. Tiengo ${ }^{1,3}$, and S. Mereghetti ${ }^{1}$ \\ 1 INAF, Istituto di Astrofisica Spaziale e Fisica Cosmica - Milano, via Bassini 15, 20133 Milano, Italy \\ e-mail: nicola@iasf-milano.inaf.it \\ 2 INAF, Osservatorio Astronomico di Cagliari, località Poggio dei Pini, strada 54, 09012 Capoterra, Italy \\ ${ }^{3}$ IUSS-Istituto Universitario di Studi Superiori, viale Lungo Ticino Sforza 56, 27100 Pavia, Italy
}

Received 7 October 2011 / Accepted 16 December 2011

\section{ABSTRACT}

\begin{abstract}
Many X-ray accreting pulsars have a soft excess below $10 \mathrm{keV}$. This feature was also detected in faint sources and at low luminosity levels, suggesting that it is an ubiquitous phenomenon. For the high luminosity pulsars $\left(L_{\mathrm{X}}>10^{36} \mathrm{erg} \mathrm{s}^{-1}\right)$, the fit to this component with thermal emission models usually provides low temperatures $(k T<0.5 \mathrm{keV})$ and large emission regions $(R \geq$ a few hundred km), hence it is referred to as a "soft" excess. Nevertheless, we previously found that in persistent, low-luminosity $\left(L_{\mathrm{X}} \sim 10^{34}\right.$ erg s $\left.\mathrm{s}^{-1}\right)$ and long-period $(P>100 \mathrm{~s})$ Be accreting pulsars the observed excess can be modeled with a rather hot $\left(k T_{\mathrm{BB}}>1 \mathrm{keV}\right)$ black-body component of small area $\left(R_{\mathrm{BB}}<0.5 \mathrm{~km}\right)$, which can be interpreted as emission from the NS polar caps. In this paper, we present an analysis of an XMM-Newton observation of the Galactic Be pulsar RX J0440.9+4431, which is a poorly studied member of this class of sources. We find a best-fit period of $P=204.96 \pm 0.02 \mathrm{~s}$, which implies an average pulsar spin-down over the past 13 years of $\dot{P} \simeq 6 \times 10^{-9} \mathrm{~s} \mathrm{~s}^{-1}$. The estimated source luminosity is $L_{\mathrm{X}} \sim 8 \times 10^{34} \mathrm{erg} \mathrm{s}^{-1}$, which is higher by a factor of less than ten compared to those obtained in the first source observations, but almost two orders of magnitude lower than those measured during the few outbursts that have been detected most recently. The source spectrum can be described with a power-law and black-body model, with $k T_{\mathrm{BB}}=1.34 \pm 0.04 \mathrm{keV}$ and $R_{\mathrm{BB}}=273 \pm 16 \mathrm{~m}$, suggesting a polar-cap origin of this component. Our results support the classification of RX J0440.9+4431 as a persistent Be/NS pulsar, and confirm that the hot black-body spectral component is a common property of this class of sources.
\end{abstract}

Key words. X-rays: binaries - accretion, accretion disks - stars: emission line, Be - pulsars: individual: LS V +44 17 X-rays: individuals: RX J0440.9+4431

\section{Introduction}

Most of the X-ray binary pulsars (XBPs) are high mass X-ray binaries (HMXRBs) in which a neutron star (NS) with magnetic field $B \sim 10^{12} \mathrm{G}$ is accreting matter from a high-mass early-type star that is either an OB supergiant or a Be star. They can be persistently bright, with luminosities in excess of $10^{34} \mathrm{erg} \mathrm{s}^{-1}$, or transient sources characterized by quiescent phases, with emission around $10^{34} \mathrm{erg} \mathrm{s}^{-1}$ or less, interrupted by bright outbursts reaching $L_{X} \sim 10^{36-38} \mathrm{erg} \mathrm{s}^{-1}$ (Negueruela 1998; Reig 2007; Sidoli 2010).

In these sources, the X-ray spectra between 0.1 and $10 \mathrm{keV}$ are usually described by a rather flat power-law, with a photon index of about one, but several XBPs have shown a marked "soft" X-ray excess above the main power-law component (see La Palombara \& Mereghetti 2006, for a review). This excess is well-described by a thermal emission model (either blackbody, bremsstrahlung, or mekal) with low temperature $\left(k T_{\mathrm{SE}}<\right.$ $0.5 \mathrm{keV})$ and large emission area $\left(R_{\mathrm{SE}} \geq \mathrm{a}\right.$ few hundred $\left.\mathrm{km}\right)$. This feature has been detected not only in the high-luminosity sources (with $L_{\mathrm{X}} \sim 10^{37-38} \mathrm{erg} \mathrm{s}^{-1}$ ) but also in several low-luminosity $\left(L_{\mathrm{X}} \sim 10^{35-36} \mathrm{erg} \mathrm{s}^{-1}\right)$ XBPs observed in the Small Magellanic Cloud (SMC), where its detection is made easier by the low interstellar absorption (Sasaki et al. 2003; Ueno et al. 2004; Majid et al. 2004; Haberl \& Pietsch 2005; Haberl et al. 2008). Only in a few cases has this low-energy component showed coherent pulses and the debate about its origin remains open. Hickox et al. (2004) demonstrated that a soft spectral component could be a very common, if not ubiquitous, feature intrinsic to X-ray pulsars: it is visible in all sources with a sufficiently high flux and small absorption, and its origin is related to the source total luminosity.

On the basis of XMM-Newton data, we also observed a clear thermal excess in three of the four persistent $\mathrm{Be}$ pulsars originally identified by Reig \& Roche (1999), i.e. RX J0146.9+6121/LS I +61 ${ }^{\circ} 235$ (La Palombara \& Mereghetti 2006), 4U 0352+309/X Persei (La Palombara \& Mereghetti 2007), and RX J1037.5-5647/LS 1698 (La Palombara et al. 2009). These three sources have a persistently low luminosity $\left(L_{\mathrm{X}} \sim 10^{34-35} \mathrm{erg} \mathrm{s}^{-1}\right)$ and a long pulse period $(P>100 \mathrm{~s})$. These properties suggest that the NS orbits the Be star in a wide and nearly circular orbit, continuously accreting material from the low-density outer regions of the circumstellar envelope; in the case of $4 \mathrm{U} 0352+309$, this picture is supported by the long orbital period of 250.3 days (Delgado-Martí et al. 2001). For these sources, the detection of the thermal component was made easier by the small distance $(d \leq 5 \mathrm{kpc})$ and interstellar absorption $\left(N_{\mathrm{H}} \sim 10^{21} \mathrm{~cm}^{-2}\right)$. We found that their soft excess can only be fitted with a black-body (other simple models being rejected) that contributes $30-40 \%$ of the total flux. Interestingly, in comparison with the other, more luminous sources, their black-body component is characterized by a higher temperature $\left(k T_{\mathrm{BB}}>1 \mathrm{keV}\right)$ and a much smaller emission radius $\left(R_{\mathrm{BB}}<0.5 \mathrm{~km}\right)$. This hot $B B$ spectral component sets these low-luminosity and long-period sources apart from all the other 
pulsars, strongly suggesting that they form a distinct class. In their case, the thermal component could be produced by a different emission mechanism than in the high-luminosity pulsars. On the basis of the study of Hickox et al. (2004), it can be attributed to emission from the neutron-star polar caps. This idea is supported by the emission area of the black-body component, which is consistent with the estimated polar cap size, and the low energy part of the spectrum being clearly pulsed.

In this paper, we present the results of an XMM-Newton observation of RX J0440.9+4431, an example of this class of $\mathrm{Be} / \mathrm{NS}$ pulsars. This system was discovered by the ROSAT Galactic plane survey (Motch et al. 1997) and identified with LS V +44 17, a moderately reddened $(E(B-V)=0.65 \pm 0.05)$ B0.2 Ve star at $\sim 3.3 \mathrm{kpc}$ (Reig 2011). Thanks to observations with the PCA instrument on board RossiXTE, Reig \& Roche (1999) performed the first detailed timing and spectral analysis, and discovered a pulsation with period $P=202.5 \pm 0.5 \mathrm{~s}$. Its spectrum was accurately fitted with different models (powerlaw, power-law plus black-body, two black-bodies, and cut-off power-law) and the measured flux implied that it has a luminosity of $3 \times 10^{34} \mathrm{erg} \mathrm{s}^{-1}$ between 3 and $30 \mathrm{keV}$. RX J0440.9+4431 was also detected in the hard $\mathrm{X}$-ray range, and was reported (as source PBC J0440.9+4432) in the Palermo Swift-BAT hard X-ray catalogue (Cusumano et al. 2010), with a 15-150 keV flux of $(2.0 \pm 1.1) \times 10^{-11} \mathrm{erg} \mathrm{cm}^{-2} \mathrm{~s}^{-1}$, and in the catalogue obtained with the INTEGRAL/IBIS 7-year All-Sky Hard X-ray Survey (Krivonos et al. 2010a), with a 17-60 keV flux of $(1.36 \pm 0.22) \times 10^{-11} \mathrm{erg} \mathrm{cm} \mathrm{cm}^{-2} \mathrm{~s}^{-1}$.

In the optical/IR waveband, the $\mathrm{H} \alpha$ line has a double-peak profile, varying from a symmetric shape to a completely distorted one on one side (V/R phases), with a correlation between the equivalent width of the $\mathrm{H} \alpha$ line and the infrared magnitudes such that as the $\mathrm{EW}(\mathrm{H} \alpha)$ decreases the IR magnitudes become fainter. This long-term optical/IR variability is attributed to structural changes in the Be star's circumstellar disc, which alternates between decline and recovery phases on typical timescales of $T_{\text {disc }}>10$ yrs (Reig et al. 2005).

During the RossiXTE observation, this pulsar displayed little X-ray variability (by a factor $<10$ ), thus it was included in the class of persistent, low-luminosity and long-period Be pulsars. However, this source has since experienced three consecutive flux increases, separated by about five months from each other: the first one was detected by MAXI/GSC on 31 March 2010 (Morii et al. 2010) and by RossiXTE on 6 April 2010 (Finger \& Camero-Arranz 2010); the second one was observed by INTEGRAL on 1 September 2010 (Krivonos et al. 2010b); the third one was seen by Swift on 29 January 2011 (Tsygankov et al. 2011). The mean time between the starts of these outbursts was $\sim 155$ days, and the peak luminosities of these flares were lower than $10^{37} \mathrm{erg} \mathrm{s}^{-1}$ (assuming a source distance of $3.3 \mathrm{kpc}$ ). Assuming that these events occurred at the periastron passage of the NS, where it approaches the decretion disc of the Be star, their separation is a good estimate of the orbital period of the system. We note that the value of 155 days is also in agreement with the period of about 150 days derived from the Corbet diagram of $P_{\text {spin }}$ versus $P_{\text {orbit }}$ (Corbet 1986). Therefore, after $4 \mathrm{U} 0352+308 / \mathrm{X}$ Persei, RX J0440.9+4431 could be the second persistent $\mathrm{Be} / \mathrm{X}$-ray pulsar with a known orbital period.

\section{Observations and data reduction}

RX J0440.9+4431 was observed with XMM-Newton on 2011 March 18 (MJD = 55638.417). The three EPIC cameras, i.e. one pn (Strüder et al. 2001) and two MOS (Turner et al. 2001),

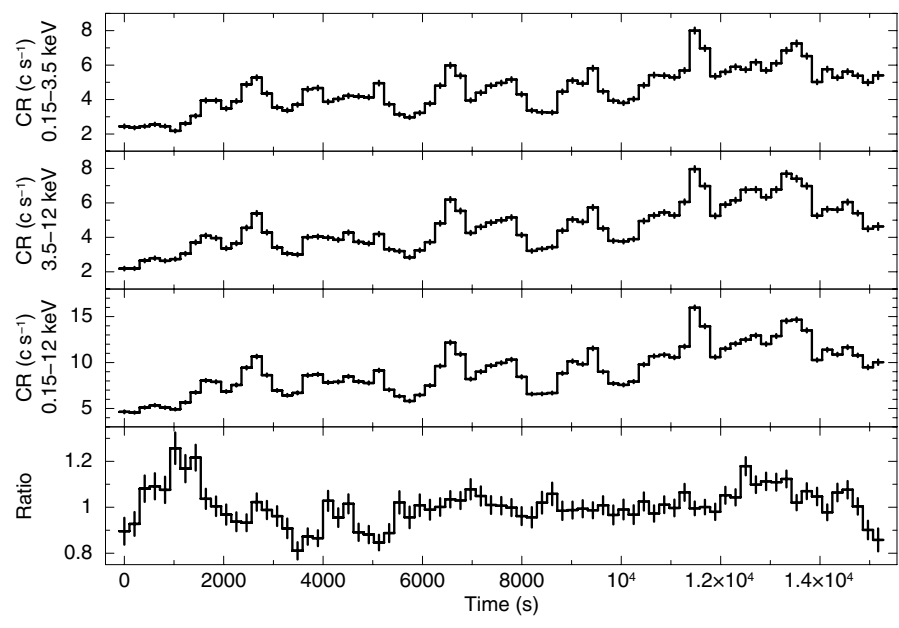

Fig. 1. Background-subtracted light curves of RX J0440.9+4431 in the energy ranges $0.15-3.5,3.5-12$, and $0.15-12 \mathrm{keV}$, with a time bin of 205 s (i.e. one pulse period).

were operated in large window mode, with a time resolution of $48 \mathrm{~ms}$ for the $p n$ camera and of $0.9 \mathrm{~s}$ for the two MOS cameras; the effective source exposure time was, respectively, of $\sim 14 \mathrm{ks}$ and $\sim 17 \mathrm{ks}$. For all cameras the medium thickness filter was used. RX J0440.9+4431 was also observed for $\sim 17 \mathrm{ks}$ by the Reflection Grating Spectrometer (RGS), which was operated in spectroscopy mode (den Herder et al. 2001).

We used version 11.0 of the XMM-Newton Science Analysis System (SAS) to process the event files. After the standard pipeline processing, we searched for possible intervals of high instrumental background, with a negative result. EPIC source events were selected within a circular area, with an extraction radius of $30^{\prime \prime}$ for all the cameras; the corresponding background events were accumulated on large circular areas free of sources and with radii of $120^{\prime \prime}, 50^{\prime \prime}$, and $60^{\prime \prime}$ for the $p n$, MOS1, and MOS2 cameras, respectively. We selected all the events in the energy range $0.15-12 \mathrm{keV}$, with pattern range $0-4$ (i.e. monoand bi-pixel events) for the $p n$ camera and 0-12 (i.e. from 1 to 4 pixel events) for the two MOS. In all cases, the background contribution to the total count rate $(\mathrm{CR})$ was negligible, resulting in a net CR of $\sim 5.8 \mathrm{cts} \mathrm{s}^{-1}$ for the $p n$ and $\sim 1.8 \mathrm{cts} \mathrm{s}^{-1}$ for each of the two MOS. Although the source CR was very high, we checked with the SAS task epatplot that no event pile-up affected our data.

\section{Timing analysis}

For the timing analysis, we considered only the EPIC data. To measure the pulse period, we converted the event arrival times to the solar system barycenter and combined the three datasets. We measured the pulse period by a standard phase-fitting technique (Dall'Osso et al. 2003), obtaining a best-fit period of $P=204.96 \pm 0.02 \mathrm{~s}$. This result takes into account the effects of the source variability during the observation (Fig. 1); the individual pulses are indeed detected with high significance throughout the entire observation, even when the source is faint.

In Fig. 1 we report the background-subtracted light curves in the energy ranges $0.15-3.5$ (soft), 3.5-12 (hard), and 0.15-12 keV (total), together with the hardness-ratio (HR) of the hard $(\mathrm{H})$ to soft $(\mathrm{S})$ light curves (computed as $\mathrm{H} / \mathrm{S}$ ); the two energy ranges were defined in order to obtain a comparable number of counts, while the time bin of $205 \mathrm{~s}$, corresponding to one 


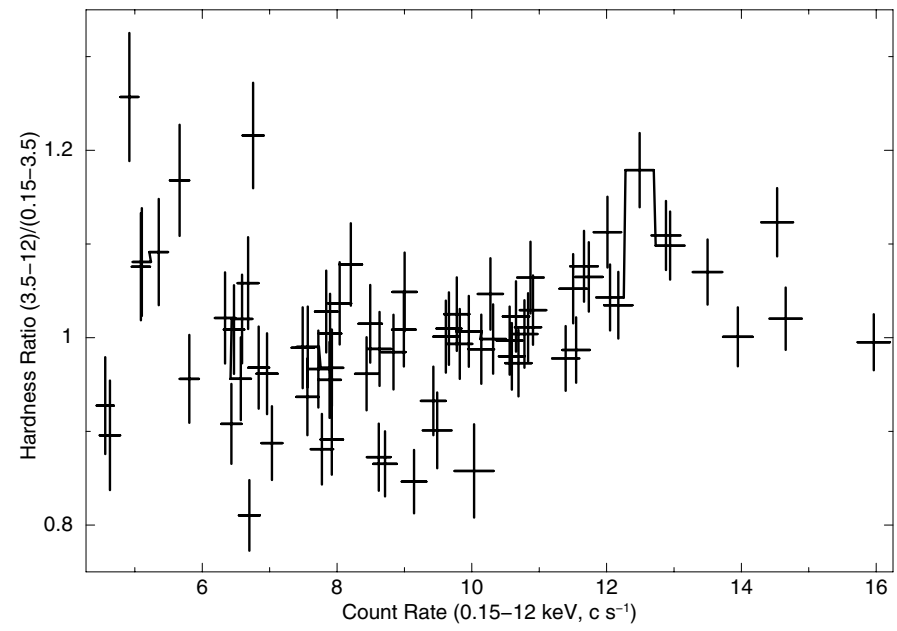

Fig. 2. Hardness-ratio variation of RX J0440.9+4431 as a function of the $0.15-12 \mathrm{keV}$ count rate, with a time bin of $205 \mathrm{~s}$.

pulse period, was chosen to avoid the effects of the periodic pulsations. In both ranges, the average $\mathrm{CR}$ is $\sim 4.5 \mathrm{cts} \mathrm{s}^{-1}$, with an increasing trend with time during the observation; moreover, the CR was highly variable over the short timescale, since there are CR variations of up to $\sim 30 \%$ between consecutive time bins. In addition the HR is characterized by a similar variability but, in this case, no long time-scale trend is observed; there is also no clear correlation with the source CR (Fig. 2), although the fit with a constant value $\mathrm{HR}=1$ is rejected $\left(\chi_{v}^{2} /\right.$ d.o.f. $\left.=3.62 / 74\right)$.

The $X M M-N e w t o n$ observation allowed us to investigate the pulse profile, for the first time, even at energies below $3 \mathrm{keV}$. In Fig. 3, we show the folded light curves in four different energy ranges, defined in order to obtain a comparable number of counts in each of them, together with the hardness ratio of the 3.4-10 to $0.15-3.4 \mathrm{keV}$ energy ranges. The shape of the pulse profile is similar in the four ranges: in all cases, it shows a single broad peak, but it is impossible to fit it with a simple sinusoidal model; the measured pulsed fraction, defined as $\left(\mathrm{CR}_{\max }-\mathrm{CR}_{\min }\right) /(2 \times$ $\mathrm{CR}_{\text {average }}$ ), is $\sim 55 \%$ for all the energy ranges; the increasing part of the curve is more regular than the decreasing one, which is steeper at the beginning and flatter at the end. However, we note an energy dependence of the pulse profile around the CR minimum (phase $\phi=0.7$ ): the minimum CR value is reached at slightly later phases for increasing energies, and also the following CR increase is delayed; moreover, at the minimum egress the $\mathrm{CR}$ increases more suddenly at high energies than at the low ones. As a consequence, the HR of the hard to soft curves is highly variable in this phase interval: it first shows a sharp minimum just after the CR minimum, suddenly followed by a large peak.

\section{Spectral analysis}

For the EPIC source and background spectra, we adopted the same extraction parameters used for the light curves; we generated the applicable response matrices and ancillary files using the SAS tasks rmfgen and arfgen. We also considered the RGS data, using the source and background spectra and the response matrices obtained with the standard reduction pipeline. To ensure the applicability of the $\chi^{2}$ statistics, the EPIC and RGS spectra were rebinned with a minimum of 100 and 30 counts per bin, respectively; they were then fitted in the energy range $0.3-12 \mathrm{keV}$ using XSPEC 12.4.0. In the following,

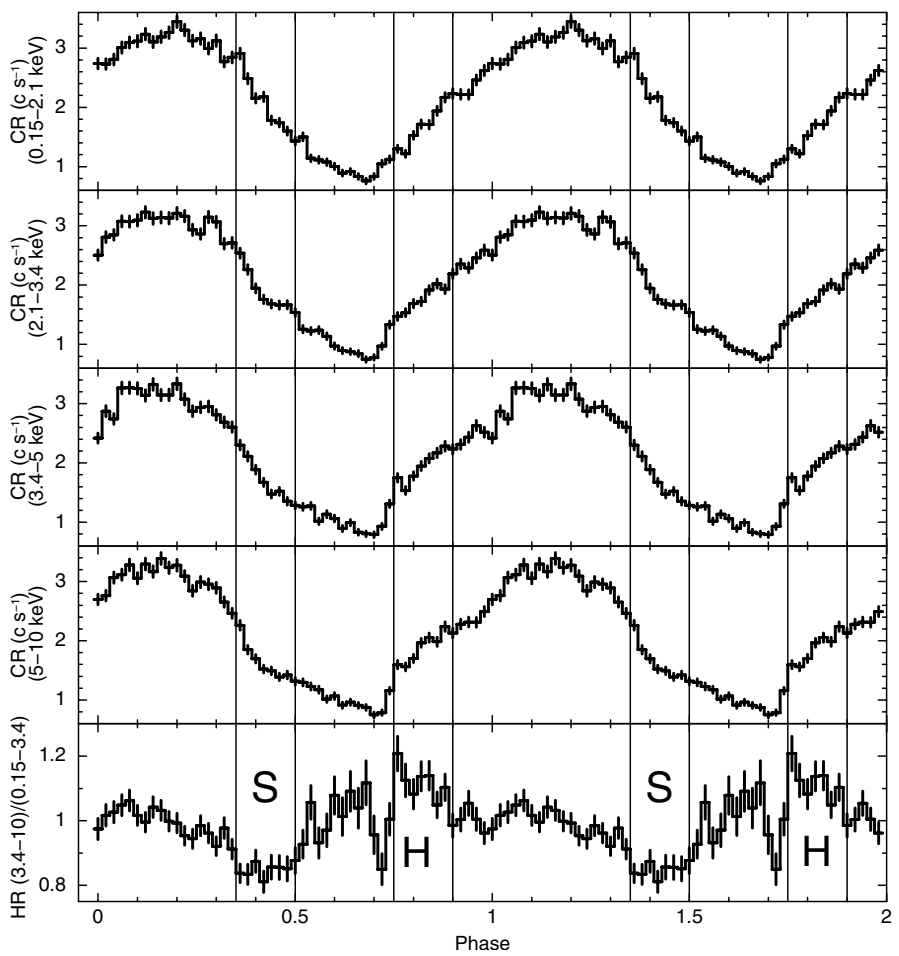

Fig. 3. Background-subtracted light curves of RX J0440.9+4431 in the energy ranges $0.15-2.1,2.1-3.4,3.4-5$, and $5-10 \mathrm{keV}$, folded at the bestfit period $P=204.96 \mathrm{~s}$.

all spectral uncertainties and upper limits are given at the $90 \%$ confidence level for one interesting parameter, and we assume a source distance of $3.3 \mathrm{kpc}$.

After checking that separate fits of the three EPIC cameras gave consistent results, we fitted them simultaneously to improve the quality of the statistics; we then checked that both RGS spectra were consistent with the EPIC ones, thus also included them in the spectral analysis; to this aim, we introduced relative normalization factors among the spectra of the five cameras. Using an absorbed power-law $(P L)$ model, we obtained a hydrogen column density $N_{\mathrm{H}}=(1.19 \pm 0.02) \times 10^{22} \mathrm{~cm}^{-2}$ and a photon-index $\Gamma=1.16 \pm 0.01$, with $\chi_{v}^{2} /$ d.o.f. $=2.097 / 1083$. Using an absorbed black-body $(B B)$ model, we obtained $N_{\mathrm{H}}=$ $(3.33 \pm 0.0 .9) \times 10^{21} \mathrm{~cm}^{-2}, \mathrm{a} B B$ temperature $k T_{\mathrm{BB}}=1.58 \pm$ $0.01 \mathrm{keV}$, and a $B B$ radius $R_{\mathrm{BB}}=306 \pm 3 \mathrm{~m}$, with $\chi_{v}^{2} /$ d.o.f. $=$ $2.604 / 1083$.

In both cases, the fits of the spectrum were unacceptable, with large residuals, so we repeated the fit with a $P L+B B$ model. In this way, we obtained a significant improvement (Fig. 4), with $\chi_{v}^{2} /$ d.o.f. $=1.105 / 1081$. The corresponding best-fit parameters are $N_{\mathrm{H}}=(7.3 \pm 0.4) \times 10^{21} \mathrm{~cm}^{-2}, \Gamma=0.85 \pm 0.07$, and $k T_{\mathrm{BB}}=1.34 \pm 0.04 \mathrm{keV}$. The $P L$ normalization is $I_{\mathrm{PL}}=(1.8 \pm$ $0.2) \times 10^{-3} \mathrm{ph} \mathrm{cm}^{-2} \mathrm{~s}^{-1} \mathrm{keV}^{-1}$ at $1 \mathrm{keV}$ and the $B B$ radius $R_{\mathrm{BB}}=$ $273 \pm 16 \mathrm{~m}$. The absorbed flux in the energy range $0.3-12 \mathrm{keV}$ is $f_{\mathrm{abs}, \mathrm{X}}=6.0_{-0.3}^{+0.2} \times 10^{-11} \mathrm{erg} \mathrm{cm}^{-2} \mathrm{~s}^{-1}$, while the corresponding unabsorbed flux is $f_{\text {unabs, } \mathrm{X}}=6.7 \times 10^{-11} \mathrm{erg} \mathrm{cm}^{-2} \mathrm{~s}^{-1}$, which implies a source luminosity $L_{X}=8.3 \times 10^{34} \mathrm{erg} \mathrm{s}^{-1}$. The $B B$ component contributes to about $35 \%$ of the $0.3-12 \mathrm{keV}$ source flux.

Neither the EPIC nor the RGS spectra have clear emission or absorption features. We also searched for narrow iron $K_{\alpha}$ emission lines between 6 and $7 \mathrm{keV}$, with different widths between 0 and $0.5 \mathrm{keV}$. We found no evidence of these lines, with an upper limit on their equivalent widths of $\sim 70 \mathrm{eV}$ (at 90\% c.l.) at most. 


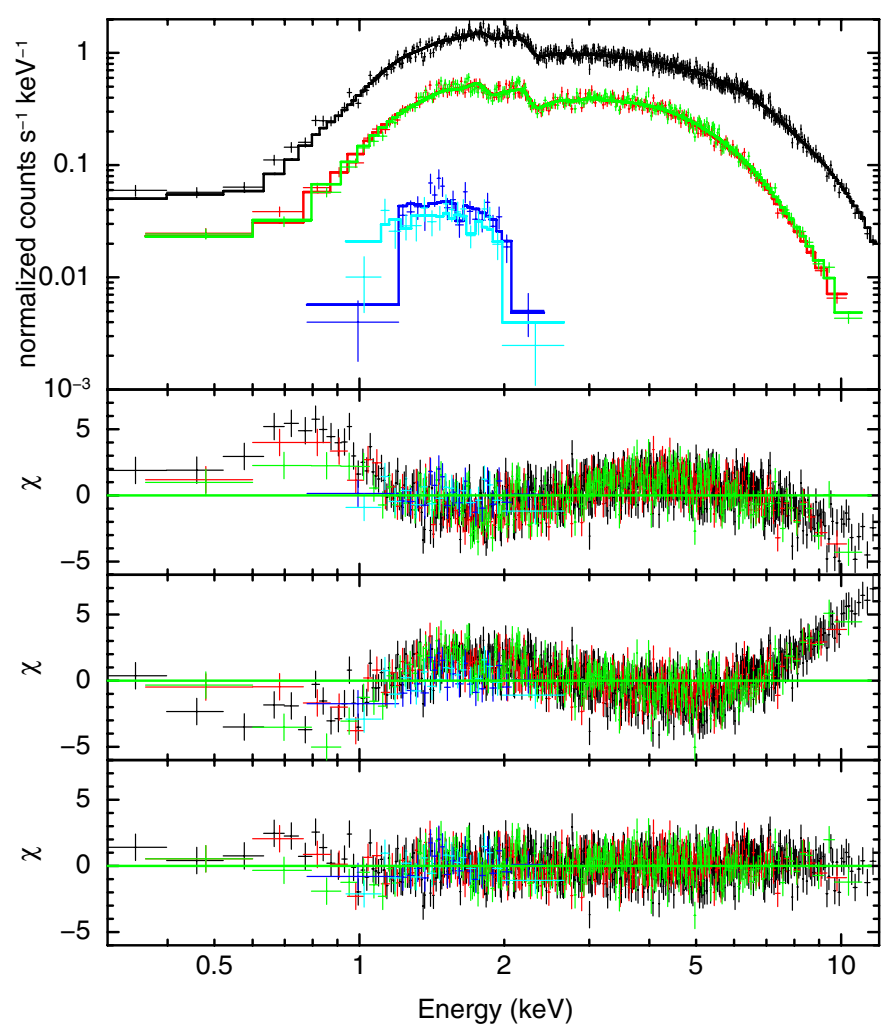

Fig. 4. Top panel: total spectrum of RX J0440.9+4431 with the best-fit $P L+B B$ model. The spectra of the $p n, \operatorname{MOS} 1$ and MOS2 cameras are shown in black, red, and green, respectively, while those of the RGS1 and $R G S 2$ are shown in blue and light blue. Middle panels: residuals (in units of $\sigma$ ) between data and model in the case of the single $P L$ and of the single $B B$. Bottom panel: residuals in the case of the $P L+B B$.

\section{Phase-resolved spectroscopy}

As shown in Fig. 3, the HR is characterized by large variations along the pulse, hence it is interesting to investigate the spin-phase resolved spectrum. To this aim, we first analyzed the background-subtracted spectra of the EPIC data in two different phase intervals, i.e. the soft $(\mathrm{S}, \phi=0.35-0.50)$ and the hard $(\mathrm{H}$, $\phi=0.75-0.90)$ : in spite of the HR difference, they are characterized by a comparable CR in the total $(0.15-12)$ energy range and, then, of photon counts in the accumulated spectra.

The independent fits of the two spectra with a single $P L$ or $B B$ model do not provide satisfactory results (in some cases they are rejected by the data), while the use of a $P L+B B$ model significantly improves the spectral fit goodness; the best-fit parameters are reported in Table 1. Taking into account the estimated uncertainties, none of the spectral parameters vary significantly between the $\mathrm{S}$ and the $\mathrm{H}$ spectra: in all cases, they are characterized by comparable or consistent best-fit values.

We also investigated the spectral variability at the pulse minimum, where the HR shows an abrupt variation, by considering the spectrum of two narrow phase ranges at $\phi=0.72$ and $\phi=0.76$. Owing to the limited count statistics, it was impossible to perform a detailed spectral analysis, but we checked that the spectra in these regions are consistent with the best-fit models of the $\mathrm{S}$ and $\mathrm{H}$ phase intervals, respectively.

To investigate the relative variations in the two components with the period phase, we also simultaneously fitted the $S$ and $\mathrm{H}$ spectra with the $P L+B B$ model by assuming the same value of $N_{\mathrm{H}}, \Gamma$, and $k T_{\mathrm{BB}}$ for the two phase intervals. In this case,
Table 1. Best-fit spectral parameters for the phase-resolved spectroscopy of RX J0440.9+4431, in the case of the independent fit of the two spectra.

\begin{tabular}{lcc}
\hline \hline Phase interval & $\mathrm{S}$ & $\mathrm{H}$ \\
\hline$N_{\mathrm{H}}\left(\times 10^{22} \mathrm{~cm}^{-2}\right)$ & $0.76_{-0.14}^{+0.13}$ & $0.69_{-0.17}^{+0.16}$ \\
\hline$\Gamma$ & $1.12_{-0.24}^{+0.26}$ & $0.89_{-0.42}^{+0.35}$ \\
$f_{\mathrm{PL}}{ }^{a}$ & $2.76_{-0.31}^{+0.08}(63 \%)$ & $2.28_{-0.30}^{+0.19}(44 \%)$ \\
\hline$k T_{\mathrm{BB}}(\mathrm{keV})$ & $1.35_{-0.13}^{+0.17}$ & $1.54_{-0.11}^{+0.12}$ \\
$R_{\mathrm{BB}}(\mathrm{m})$ & $237_{-37}^{+49}$ & $247_{-27}^{+36}$ \\
$f_{\mathrm{BB}}{ }^{a}$ & $1.63_{-0.05}^{+0.25}(37 \%)$ & $2.95_{-0.15}^{+0.91}(56 \%)$ \\
\hline$f_{\mathrm{TOT}}{ }^{a}$ & $4.39_{-0.42}^{+0.18}$ & $5.23_{-1.25}^{+0.22}$ \\
$\chi_{v}^{2} /$ d.o.f. & $1.15 / 153$ & $1.09 / 170$ \\
\hline
\end{tabular}

Notes. ${ }^{(a)}$ Absorbed flux in the energy range $0.3-12 \mathrm{keV}$, in units of $10^{-11} \mathrm{erg} \mathrm{cm}^{-2} \mathrm{~s}^{-1}$.

we obtained $N_{\mathrm{H}}=\left(0.92_{-0.07}^{+0.08}\right) \times 10^{22} \mathrm{~cm}^{-2}, \Gamma_{\mathrm{PL}}=1.48_{-0.13}^{+0.17}$, and $k T_{\mathrm{BB}}=1.67 \pm 0.05 \mathrm{keV}$, with $\chi_{v}^{2} /$ d.o.f. $=1.17 / 328$. The corresponding normalization values are reported in Table 2 (Case 1). With these constraints on the model parameters, the spectral changes as a function of the phase are reproduced by the variations in the relative contribution of the two components. The values reported in Table 2 show that, with this assumption, the observed spectral variability is due to both components, since their flux varies by more than $50 \%$ between the two phases, even if in opposite directions: the $P L$ flux decreases and the $B B$ flux increases from the $\mathrm{S}$ to the $\mathrm{H}$ phase.

To confirm that the thermal component varies as a function of the rotational phase, we should prove that a constant $B B$ component is rejected by the data. To this aim, we modified the test model by linking the $B B$ parameters for the two spectra together, while both the photon-index $\Gamma$ and the normalization $I_{\mathrm{PL}}$ of the $P L$ component were able to vary independently in the two phase intervals. The resulting best-fit model implies that $N_{\mathrm{H}}=(0.84 \pm 0.09) \times 10^{22} \mathrm{~cm}^{-2}, k T_{\mathrm{BB}}=1.54 \pm 0.08 \mathrm{keV}$, and $R_{\mathrm{BB}}=223_{-16}^{+17} \mathrm{~m}$. The power-law parameters are shown in Table 2 (Case 2). Even with this model, we found a good quality fit $\left(\chi_{v}^{2} /\right.$ d.o.f. $\left.=1.16 / 328\right)$, that is fully comparable to the previous case. This result suggests that it is possible to attribute the whole spectral variability to the $P L$ component, and that a constant $B B$ cannot be ruled out.

\section{Discussion}

Our XMM-Newton observation of RX J0440.9+4431 is the first of this source performed with a large X-ray telescope of the last generation, and has allowed us to investigate, for the first time, the timing and spectral properties of this pulsar at low X-ray energies below $3 \mathrm{keV}$.

We have obtained a new measurement of the pulse period: $P=204.96 \pm 0.02 \mathrm{~s}$. This value agrees with the period measurements obtained by the Gamma-ray Burst Monitor on-board the FERMI satellite since its launch in $2008^{1}$, and collectively these data demonstrate that this source is characterized by a variable spin period, that is typical of wind-fed binary systems. On a longer timescale, our value is slightly larger than the previous value of $202.5 \pm 0.5 \mathrm{~s}$ found in 1998 by RossiXTE (Reig \& Roche 1999): this period difference $(\triangle P / P \simeq 1 \%)$ is too large to be ascribed to orbital motion and implies that the average

\footnotetext{
1 http://gammaray.nsstc .nasa.gov/gbm/science/pulsars/ lightcurves/rxj0440_fig1.png
} 
Table 2. Best-fit values for the black-body and power-law parameters, when the two spectra are fitted simultaneously with common values of $N_{\mathrm{H}}$, $\Gamma$, and $k T_{\mathrm{BB}}\left(\right.$ Case 1) or with common values of $N_{\mathrm{H}}, k T_{\mathrm{BB}}$, and $R_{\mathrm{BB}}($ Case 2).

\begin{tabular}{lcccc}
\hline \hline Phase & \multicolumn{2}{c}{ Case 1 } & \multicolumn{2}{c}{ Case 2 } \\
interval & $\mathrm{S}$ & $\mathrm{H}$ & $\mathrm{S}$ & $\mathrm{H}$ \\
\hline$N_{\mathrm{H}}{ }^{a}$ & $0.92_{-0.07}^{+0.08}$ & $0.92_{-0.07}^{+0.08}$ & $0.84 \pm 0.09$ & $0.84 \pm 0.09$ \\
\hline$\Gamma$ & $1.48_{-0.13}^{+0.17}$ & $1.48_{-0.13}^{+0.17}$ & $1.52_{-0.19}^{+0.22}$ & $1.00_{-0.15}^{+0.16}$ \\
$I_{\mathrm{PL}}{ }^{b}$ & $3.37_{-0.45}^{+0.55}$ & $2.24_{-041}^{+0.49}$ & $2.65_{-0.59}^{+0.68}$ & $1.83_{-0.40}^{+0.44}$ \\
$f_{\mathrm{PL}}{ }^{c}$ & $2.34_{-0.42}^{+0.28}(54 \%)$ & $1.56_{-0.22}^{+0.09}(31 \%)$ & $1.73_{-0.28}^{+0.10}(42 \%)$ & $2.93_{-0.26}^{+0.06}(55 \%)$ \\
\hline$k T_{\mathrm{BB}}$ & $1.67 \pm 0.05$ & $1.67 \pm 0.05$ & $1.54 \pm 0.08$ & $1.54 \pm 0.08$ \\
$R_{\mathrm{BB}}$ & $176_{-14}^{+13}$ & $231_{-13}^{+12}$ & $223_{-16}^{+17}$ & $223_{-16}^{+17}$ \\
$f_{\mathrm{BB}}{ }^{a}$ & $2.02_{-0.10}^{+0.07}(46 \%)$ & $3.47_{-0.06}^{+0.05}(69 \%)$ & $2.42_{-0.10}^{+0.06}(58 \%)$ & $2.42_{-0.10}^{+0.06}(45 \%)$ \\
\hline$f_{\mathrm{TOO}}{ }^{a}$ & $4.36_{-0.16}^{+0.10}$ & $5.03_{-0.11}^{+0.14}$ & $4.15_{-0.20}^{+0.12}$ & $5.35_{-0.28}^{+0.20}$ \\
$\chi_{v}^{2}$ & \multicolumn{3}{c}{1.16} \\
d.o.f. & \multicolumn{3}{c}{328} \\
\hline
\end{tabular}

Notes. ${ }^{(a)} \times 10^{22} \mathrm{~cm}^{-2}{ }^{(b)} \times 10^{-3} \mathrm{ph} \mathrm{cm}^{-2} \mathrm{~s}^{-1} \mathrm{keV}^{-1}$ at $1 \mathrm{keV}$; ${ }^{(c)}$ absorbed flux in the energy range $0.3-12 \mathrm{keV}$, in units of $10^{-11} \mathrm{erg} \mathrm{cm}^{-2} \mathrm{~s}^{-1}$.

spin-down during the past 13 years has been $\dot{P}=(6.4 \pm 1.3) \times$ $10^{-9} \mathrm{~s} \mathrm{~s}^{-1}$.

We have estimated a source luminosity $L_{\mathrm{X}} \sim 6 \times 10^{34} \mathrm{erg} \mathrm{s}^{-1}$ in the $2-10 \mathrm{keV}$ energy range. To investigate the long-term evolution of RX J0440.9+4431, we have also considered the X-ray flux measurements obtained with ROSAT and RossiXTE. For ROSAT, we considered the CRs that were obtained during the All Sky Survey (Voges et al. 2000) and in a subsequent, specific observation (Motch et al. 1997). In their case, we used the WebPIMMS tool ${ }^{2}$ and assumed our best-fit $P L+B B$ emission model to infer the source flux in the $2-10 \mathrm{keV}$ energy band: in this way, we estimated a $2-10 \mathrm{keV}$ luminosity of, respectively, $(1.1 \pm 0.3) \times 10^{34}$ and $(2.4 \pm 0.2) \times 10^{34} \mathrm{erg} \mathrm{s}^{-1}$. For RossiXTE, we considered the value obtained with the two-blackbody model used by Reig \& Roche (1999), i.e. $\sim 2 \times 10^{34} \mathrm{erg} \mathrm{s}^{-1}$. Although RX J0440.9+4431 was observed by XMM-Newton at a higher flux level, all these luminosity estimates are in the range $10^{34}-10^{35} \mathrm{erg} \mathrm{s}^{-1}$ : the variation is smaller than a factor 10 , hence the XMM-Newton observation also suggests that RX J0440.9+4431 is a persistent BeXRB. On the other hand, a large flux increase was previously reported in three different events (Morii et al. 2010; Krivonos et al. 2010b; Tsygankov et al. 2011); based on the measured CRs, we estimated that in all cases the source luminosity had risen by up to a few $10^{36} \mathrm{erg} \mathrm{s}^{-1}$. This implies a source variability of at least two order of magnitudes, in contrast to the classification of RX J0440.9+4431 as a persistent source. In the present study, we analyzed the RossiXTE ASM light-curve data of RX J0440.9+4431 acquired since 1996, to check for the occurrence of other flux increases, but we found no clear evidence of this type of events in addition to the previous three; the flux level of RX J0440.9+4431 is therefore usually consistent with a persistent nature of the source, while the transient behavior more recently observed is likely due to structural changes in the circumstellar disc of the Be star.

From the spectral analysis, we obtained a hydrogen column density $N_{\mathrm{H}}=(7.3 \pm 0.4) \times 10^{21} \mathrm{~cm}^{-2}$, which is much lower (almost one order of magnitude) than the values estimated by RossiXTE. However, we note that the energy range of the RossiXTE spectral analysis (above $3 \mathrm{keV}$ ) is unsuitable for a robust estimate of $N_{\mathrm{H}}$, while the low-energy end of the XMM-Newton spectra $(0.3 \mathrm{keV})$ allows a much more reliable analysis. The optical counterpart of RX J0440.9+4431, LS V +4417 , has a color excess of $E(B-V)=0.65(\operatorname{Reig} 2011)$.

\footnotetext{
${ }^{2}$ http://heasarc.gsfc.nasa.gov/Tools/w3pimms_pro.html
}

Assuming $A_{\mathrm{V}}=3.1 E(B-V)$ and the average relation $A_{\mathrm{V}}=$ $N_{\mathrm{H}} \times 5.59 \times 10^{-22} \mathrm{~cm}^{-2}$ between optical extinction and X-ray absorption (Predehl \& Schmitt 1995), this implies that $N_{\mathrm{H}}=$ $3.6 \times 10^{21} \mathrm{~cm}^{-2}$, which is a factor of about two lower than our result. We note that the data used to calibrate the previous relation is characterized by a comparable dispersion, hence there is rough agreement between the two measurements.

We found evidence of a previously undetected thermal component, in addition to the main power-law: this thermal component has a high temperature $(k T=1.34 \mathrm{keV})$, a small emission area $(R=273 \mathrm{~m})$, and contributes about $35 \%$ of the source flux below $10 \mathrm{keV}$. On the other hand, it was impossible to fit the XMM-Newton spectra with the two-black-body model used by Reig \& Roche (1999). The RossiXTE spectrum showed a possible feature at $\simeq 6.2 \mathrm{keV}$, which might be a Fe-K emission line, but it was impossible to constrain its parameters, and only an upper limit of $\sim 100 \mathrm{eV}$ to the equivalent width (EQW) could be provided. In spite of the higher luminosity level, we found no evidence of the $\mathrm{Fe}-\mathrm{K}$ emission line and derived an upper limit of $\sim 70 \mathrm{eV}$ (at $90 \%$ c.1.) to its EQW.

For its size and temperature, the $B B$ excess observed in RX J0440.9+4431 is similar to those detected in the other persistent Be/NS XBPs, i.e. LS I +61 ${ }^{\circ}$ 235/RX J0146.9+6121 (La Palombara \& Mereghetti 2006), X Persei/4U 0352+309 (Coburn et al. 2001; La Palombara \& Mereghetti 2007), and LS 1698/RX J1037.5-5647 (Reig \& Roche 1999; La Palombara et al. 2009): hence, this hot BB spectral component is a common property of this type of sources. However, we note that a similar feature has also been observed in 3A $0535+262$ (Orlandini et al. 2004; Mukherjee \& Paul 2005), 4U 2206+54 (Masetti et al. 2004; Torrejón et al. 2004; Reig et al. 2009), and SAX J2103.5+4545 (İnam et al. 2004), which are other types of XBPs of low luminosity $\left(L_{\mathrm{X}} \leq 10^{35} \mathrm{erg} \mathrm{s}^{-1}\right)$ and long pulse period $(P>100 \mathrm{~s})$. Moreover, the same type of $B B$ excess has also been detected in the three supergiant fast X-ray transients (SFXTs) IGR J11215-5292 (Sidoli et al. 2007), IGR J084084503 (Sidoli et al. 2009), and XTE J1739-302 (Bozzo et al. 2010), only the first of which is a confirmed pulsar $(P=187 \mathrm{~s})$. Finally, a hot $B B$ excess has also been detected in the SMC binary pulsar SXP 1062, a possible new persistent Be X-ray binary (Hénault-Brunet et al. 2012). In Fig. 5, we report the best-fit radius and temperature for the $B B$ component of these sources, together with lines showing four different levels of the blackbody luminosity; for some source more than one set of values 


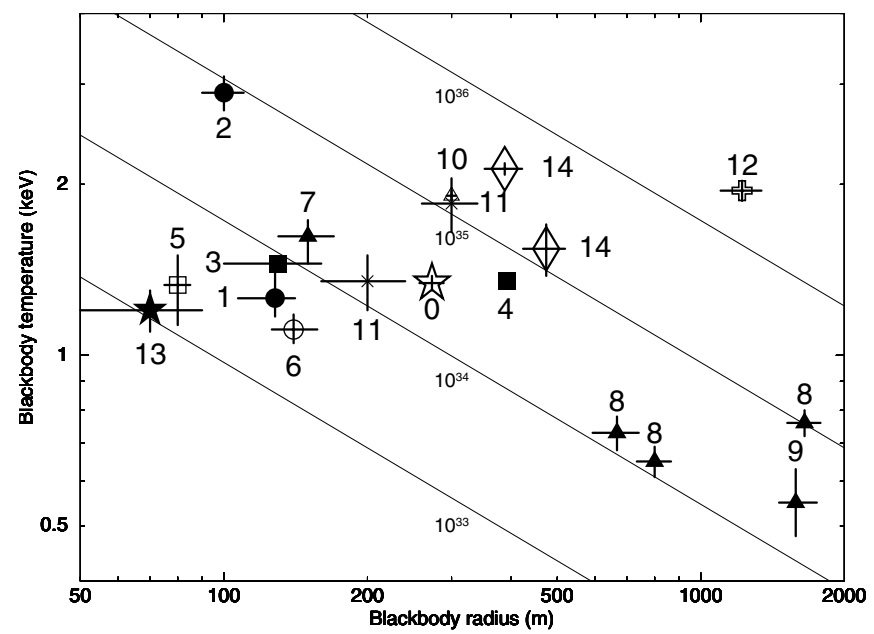

Fig. 5. Best-fit values for radius and temperature of the $B B$ component in the case of RX J0440.9+4431 (empty star), RX J1037.5-5647 (filled circles), 4U 0352+309 (filled squares), RX J0146.9+6121 (empty circle), 3A 0535+262 (empty square), SAX J2103.5+4545 (empty triangle), 4U 2206+54 (filled triangles), IGR J11215-5292 (asterisks), IGR J08408-4503 (cross), XTE J1739-302 (filled star), and SXP 1062 (empty diamonds). The continuous lines connect the black-body parameters corresponding to four different levels of luminosity (in $\mathrm{erg} \mathrm{s}^{-1}$ ). References: 0 - this work; 1- La Palombara et al. (2009); 2 - Reig \& Roche (1999); 3 - Coburn et al. (2001); 4 - La Palombara \& Mereghetti (2007); 5 - Mukherjee \& Paul (2005); 6 - La Palombara \& Mereghetti (2006); 7 - Masetti et al. (2004); 8 - Torrejón et al. (2004); 9 - Reig et al. (2009); 10 - İnam et al. (2004); 11 - Sidoli et al. (2007); 12 Sidoli et al. (2009); 13 - Bozzo et al. (2010); 14 - Hénault-Brunet et al. (2012).

is shown, corresponding to different observations or flux levels. In most cases, the spectral parameters are within a narrow range of values, i.e. $k T_{\mathrm{BB}} \sim 1-2 \mathrm{keV}$ and $R_{\mathrm{BB}}<200 \mathrm{~m}$. We emphasize that, in all these cases, the estimated total source $\mathrm{X}$-ray luminosity is $\sim 10^{34} \mathrm{erg} \mathrm{s}^{-1}$, with a $20-40 \%$ contribution of the black-body component. For RX J0440.9+4431, the $B B$ radius is slightly larger $\left(R_{\mathrm{BB}} \sim 270 \mathrm{~m}\right)$, which is consistent with its total X-ray luminosity also being higher $\left(L_{\mathrm{X}} \sim 8 \times 10^{34} \mathrm{erg} \mathrm{s}^{-1}\right)$. When observed at a high (i.e. $L_{X} \sim 10^{35} \mathrm{erg} \mathrm{s}^{-1}$ ) luminosity level, RX J1037.5-5647 (point 2), 4U 0352+309 (point 4), SAX J2103.5+4545 (point 10), IGR J11215-5292 (point 11), and SXP 1062 (point 14) also have large values of temperature and/or radius.

In contrast to this sample of sources, several XBPs are characterized by a soft excess, since the fit of this component with a thermal emission model provides low temperatures $(k T<$ $0.5 \mathrm{keV})$ and large emitting regions $(R>100 \mathrm{~km})$. In Fig. 6, we report the luminosity and pulse period of both types of XBPs: the soft excess and the hot $B B$ ones are reported as squares and circles, respectively. On the basis of their distribution in the $P-L_{\mathrm{X}}$ diagram, these pulsars are divided into two distinct groups: the sources in the first group are characterized by high luminosity $\left(L_{\mathrm{X}} \geq 10^{37} \mathrm{erg} \mathrm{s}^{-1}\right)$ and short pulse period $(P<100 \mathrm{~s})$, and in most cases they are in close binary systems with an accretion disk; those in the second group have low luminosities $\left(L_{\mathrm{X}} \leq 10^{36} \mathrm{erg} \mathrm{s}^{-1}\right)$ and long pulse periods $(P>100 \mathrm{~s})$, since they have wide orbits and are wind-fed systems. While all the pulsars in the first group have a soft excess, both types of pulsars are present in the second group. In this case, the hot $B B$ pulsars are the ones that, on average, are characterized by the lowest luminosities and the longest periods. This suggests that the hot $B B$ spectral component is a common feature of the

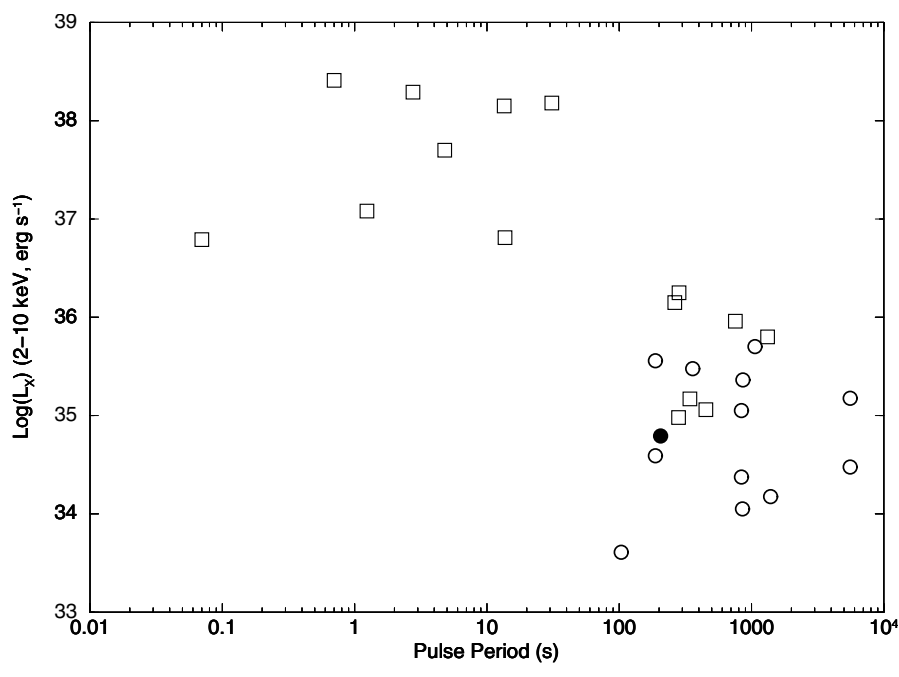

Fig. 6. X-ray luminosity (in the $2-10 \mathrm{keV}$ energy range) of the XBPs with a detected thermal excess as a function of the pulse period. The filled circle refers to the XMM-Newton observation of RX J0440.9+4431, empty circles refer to other detections of hot $B B$ pulsars, and empty squares refer to the soft excess sources.

low-luminosity and long-period XBPs. However, in the second group of sources there is no clear separation between the two types of pulsars, since there is a partial overlap between the soft excess and the hot $B B$ ones. Therefore, the origin of the spectral difference in pulsars with comparable values of luminosity and pulse period is unclear. Even if it were related to extremely different emission mechanisms operating in these sources, we cannot exclude that, at least in some cases, it is due to the degeneracy of spectral fitting in some spectra. On the basis of the X-ray spectrum it is often impossible to distinguish between a $B B$ with a small radius and high temperature and one with a large radius and low temperature (see, e.g., Bozzo et al. 2010).

On the bases of our results and the emission models proposed by Hickox et al. (2004), in the case of RX J0440.9+4431 it also makes sense to attribute the observed spectral excess to the thermal emission from the NS polar cap, in agreement with what has already been suggested for the other persistent Be pulsars. To verify this hypothesis, we assume that the source is in the "accretor" state, with matter accretion onto the NS surface. Assuming $M_{\mathrm{NS}}=1.4 M_{\odot}$ and $R_{\mathrm{NS}}=10^{6} \mathrm{~cm}$, the source luminosity $L_{\mathrm{X}}=8.3 \times 10^{34} \mathrm{erg} \mathrm{s}^{-1}$ implies an accretion rate $\dot{M} \simeq 4.5 \times 10^{14} \mathrm{~g} \mathrm{~s}^{-1}$ and, adopting $B_{\mathrm{NS}}=10^{12} \mathrm{G}$, a magnetospheric radius $R_{m} \simeq 10^{9} \mathrm{~cm}$ (Campana et al. 1998). In this case, based on the relation $R_{\mathrm{col}} \sim R_{\mathrm{NS}}\left(R_{\mathrm{NS}} / R_{m}\right)^{0.5}$ (Hickox et al. 2004), we would obtain $R_{\text {col }} \sim 320 \mathrm{~m}$. This value, which is an estimate of the expected size of the polar cap, is remarkably similar to the estimated black-body emitting radius $\left(R_{\mathrm{BB}} \sim 270 \mathrm{~m}\right)$, hence it strongly supports the idea of a polar-cap origin for the observed $B B$ emission. If this description were correct, we would expect to observe some variability in the thermal component along the pulse phase. The phase-resolved spectral analysis confirmed the spectral variability along the pulse, but this can be attributed to the $P L$ component, since a constant $B B$ component is fully compatible with the spectral data. The lack of variability of the $B B$ component does not contradict the idea of a polar-cap origin, as anisotropic radiation can appear to be steady along the pulse for some geometrical configurations of the hot spots on the NS surface (Beloborodov 2002). The phase dependence of the non-thermal component is likely due to the anisotropy of the Comptonized radiation of the accretion 
column. This was demonstrated, for example, by the use of a self-consistent model by Becker \& Wolff (2007) for the dynamics and the radiative transfer occurring in the accretion column of bright X-ray pulsars. This model is based on the physical picture originally proposed by Davidson (1973), in which the accreting gas passes through a radiative, radiation-dominated shock before settling onto the NS surface. For the relatively low luminosity of RX J0440.9+4431, the shock could be absent or have only a limited extent above the neutron star surface. Thus, the black-body emission from the dense thermal mound at the base of the column could be the dominant seed component for the Comptonization. In this geometry, the radiation is probably emerging as a "pencil beam" rather than from the column's lateral surface as in the Becker \& Wolff model.

\section{Conclusions}

We have analyzed a $\sim 17 \mathrm{ks} X M M-N e w t o n$ observation of the $\mathrm{Be} / \mathrm{NS}$ X-ray pulsar RX J0440.9+4431. The source was detected at a luminosity level $L_{X} \simeq 8.3 \times 10^{34} \mathrm{erg} \mathrm{s}^{-1}$ in the $0.3-12 \mathrm{keV}$ energy range, a luminosity that is less than one order of magnitude higher than the average level of the previous ROSAT and RossiXTE observations, hence confirms the persistent nature of this source. This classification is consistent with the large flux increases recently observed, which imply luminosity levels of a few $10^{36} \mathrm{erg} \mathrm{s}^{-1}$, since they can be explained by structural changes in the circumstellar disc of the Be mass donor.

The XMM-Newton observation has provided a refined pulse period $P=204.96 \pm 0.02 \mathrm{~s}$, which, compared to previous measurements, implies an average pulsar spin-down $\dot{P}=(6.4 \pm$ 1.3) $\times 10^{-9} \mathrm{~s} \mathrm{~s}^{-1}$ between 1998 and 2011. This is indicative of a low average accretion rate, in agreement with the persistent nature of the pulsar. The pulse profile has a complex structure, which is not sinusoidal, and at all energies the PF is $\sim 55 \%$. However, the HR is characterized by significant variations, expecially around the pulse minimum, which indicates a spectral variability of the source.

The source spectrum shows a count excess above the main power-law component, described by a black-body with $k T_{\mathrm{BB}} \simeq$ $1.3 \mathrm{keV}$ and $R_{\mathrm{BB}} \simeq 270 \mathrm{~m}$, which contributes to $\sim 35 \%$ of the source luminosity between 0.3 and $12 \mathrm{keV}$. We found no evidence of a narrow iron $K_{\alpha}$ line between 6 and $7 \mathrm{keV}$, with an upper limit of $\sim 70 \mathrm{eV}$ to its equivalent width. The blackbody radius is comparable to the estimated size of the NS polarcap, which suggests that the origin of this component is on the NS surface. The phase-resolved spectroscopy neither confirms nor disproves this scenario, since the observed spectral variability along the pulse period can be attributed to the power-law component and is consistent with a constant thermal component.

The spectral properties of RX J0440.9+4431 are in full agreement with those observed in the other three persistent Be binary pulsars 4U 0352+309, RX J0146.9+6121, and RX J1037.5-5647, thus confirming that the hot BB spectral component is a common property of this type of sources. Moreover, we have shown that the same type of feature has also been detected in other low-luminosity and long-period pulsars, hence it is an ubiquitous phenomenon that requires further investigations.

Acknowledgements. This work is based on observations obtained with $X M M-N e w t o n$, an ESA science mission with instruments and contributions directly funded by ESA Member States and NASA. We acknowledge financial contributions by the Italian Space Agency through ASI/INAF agreements $\mathrm{I} / 009 / 10 / 0$ and $\mathrm{I} / 032 / 10 / 0$ for, respectively, the data analysis and the XMM-Newton operations. P.E. acknowledges financial support from the Autonomous Region of Sardinia through a research grant under the program
PO Sardegna FSE 2007-2013, L.R. 7/2007 "Promoting scientific research and innovation technology in Sardinia".

Note added in proof. While this article was in its publication phase, we became aware of the paper of Tsygankov et al. (2012) reporting the discovery of a cyclotron absorption line in the high-energy X-ray spectrum of RX J0440.9+4431. That paper is based instead on data obtained with the INTEGRAL, Swift, and RXTE observatories during the 2010 April and September outbursts of the X-ray pulsar. Although the source during this time interval was signficantly brighter than during our XMM-Newton observation, most of the timing and spectral results described in that paper are in good agreement with our results.

\section{References}

Becker, P. A., \& Wolff, M. T. 2007, ApJ, 654, 435

Beloborodov, A. M. 2002, ApJ, 566, L85

Bozzo, E., Stella, L., Ferrigno, C., et al. 2010, A\&A, 519, A6

Campana, S., Colpi, M., Mereghetti, S., Stella, L., \& Tavani, M. 1998, A\&ARv, 8,279

Coburn, W., Heindl, W. A., Gruber, D. E., et al. 2001, ApJ, 552, 738

Corbet, R. H. D. 1986, MNRAS, 220, 1047

Cusumano, G., La Parola, V., Segreto, A., et al. 2010, A\&A, 524, A64

Dall'Osso, S., Israel, G. L., Stella, L., Possenti, A., \& Perozzi, E. 2003, ApJ, 599,485

Davidson, K. 1973, Nature, 246, 1

Delgado-Martí, H., Levine, A. M., Pfahl, E., \& Rappaport, S. A. 2001, ApJ, 546, 455

den Herder, J. W., Brinkman, A. C., Kahn, S. M., et al. 2001, A\&A, 365, L7

Finger, M. H., \& Camero-Arranz, A. 2010, The Astronomer's Telegram, 2537, 1

Haberl, F., \& Pietsch, W. 2005, A\&A, 438, 211

Haberl, F., Eger, P., \& Pietsch, W. 2008, A\&A, 489, 327

Hénault-Brunet, V., Oskinova, L. M., Guerrero, M. A., et al. 2012, MNRAS, 420, L13

Hickox, R. C., Narayan, R., \& Kallman, T. R. 2004, ApJ, 614, 881

İnam, S. Ç., Baykal, A., Swank, J., \& Stark, M. J. 2004, ApJ, 616, 463

Krivonos, R., Tsygankov, S., Revnivtsev, M., et al. 2010a, A\&A, 523, A61

Krivonos, R., Tsygankov, S., Lutovinov, A., Turler, M., \& Bozzo, E. 2010b, The Astronomer's Telegram, 2828, 1

La Palombara, N., \& Mereghetti, S. 2006, A\&A, 455, 283

La Palombara, N., \& Mereghetti, S. 2007, A\&A, 474, 137

La Palombara, N., Sidoli, L., Esposito, P., Tiengo, A., \& Mereghetti, S. 2009, A\&A, 505, 947

Majid, W. A., Lamb, R. C., \& Macomb, D. J. 2004, ApJ, 609, 133

Masetti, N., Dal Fiume, D., Amati, L., et al. 2004, A\&A, 423, 311

Morii, M., Kawai, N., Sugimori, K., et al. 2010, The Astronomer's Telegram, 2527,1

Motch, C., Haberl, F., Dennerl, K., Pakull, M., \& Janot-Pacheco, E. 1997, A\&A, 323,853

Mukherjee, U., \& Paul, B. 2005, A\&A, 431, 667

Negueruela, I. 1998, A\&A, 338, 505

Orlandini, M., Bartolini, C., Campana, S., et al. 2004, Nucl. Phys. B Proc. Supp., 132,476

Predehl, P., \& Schmitt, J. H. M. M. 1995, A\&A, 293, 889

Reig, P. 2007, MNRAS, 377, 867

Reig, P. 2011, Ap\&SS, 332, 1

Reig, P., \& Roche, P. 1999, MNRAS, 306, 100

Reig, P., Negueruela, I., Fabregat, J., Chato, R., \& Coe, M. J. 2005, A\&A, 440, 1079

Reig, P., Torrejón, J. M., Negueruela, I., et al. 2009, A\&A, 494, 1073

Sasaki, M., Pietsch, W., \& Haberl, F. 2003, A\&A, 403, 901

Sidoli, L. 2010, Am. Inst. Phys. Conf. Ser., 1314, 271

Sidoli, L., Romano, P., Mereghetti, S., et al. 2007, A\&A, 476, 1307

Sidoli, L., Romano, P., Ducci, L., et al. 2009, MNRAS, 397, 1528

Strüder, L., Briel, U., Dennerl, K., et al. 2001, A\&A, 365, L18

Torrejón, J. M., Kreykenbohm, I., Orr, A., Titarchuk, L., \& Negueruela, I. 2004, A\&A, 423, 301

Tsygankov, S., Lutovinov, A., \& Krivonos, R. 2011, The Astronomer's Telegram, 3137,1

Tsygankov, S., Krivonos, R., \& Lutovinov, A. 2012, MNRAS, accepted [arXiv: 1201.0616]

Turner, M. J. L., Abbey, A., Arnaud, M., et al. 2001, A\&A, 365, L27

Ueno, M., Yamaguchi, H., Takagi, S.-I., Yokogawa, J., \& Koyama, K. 2004, PASJ, 56, 175

Voges, W., Aschenbach, B., Boller, T., et al. 2000, VizieR Online Data Catalog, 9029, 0 